\title{
Evaluación de la calidad de vida en personas mayores y con discapacidad: la Escala Fumat
}

\section{Evaluation of the quality of life in older persons and with disability: the Fumat Scale}

\author{
L. E. GÓMEZ** \\ M. A. VERDUGO* \\ B. ARIAS** \\ P. NAVAS*
}

\begin{abstract}
RESUMEN
Este articulo tiene como objetivo el desarrollo y la validación de un instrumento específico para valorar de un modo objetivo la calidad de vida de personas mayores y personas con discapacidad que acuden a algún tipo de servicio social. La escala se desarrolló siguiendo un proceso de tres pasos: revisión de la literatura, elaboración de un banco de items y evaluación de los items seleccionados por un grupo de 15 expertos en términos de idoneidad, importancia y observabilidad. La validación del cuestionario se llevó a cabo en una muestra de 100 usuarios adultos de servicios proporcionados por Matía Fundazioa (San Sebastián). La fiabilidad se evaluó en términos de consistencia interna (Alpha de Cronbach y correlación entre dos mitades de Spearman-Brown) y estructura factorial (fiabilidad zeta y omega). La validez se evaluó en términos de validez de contenido, validez discriminante, validez concurrente y validez de constructo. Los resultados obtenidos confirman la validez y fiabilidad de la Escala FUMAT para evaluar la calidad de vida objetiva de personas mayores y personas con discapacidad que son usuarias de servicios sociales.
\end{abstract}

\section{PALABRAS CLAVE}

Evaluación de la calidad de vida, Personas mayores, Discapacidad, Evaluación, Medida, Psicometría.

\footnotetext{
* INICO. Universidad de Salamanca.

** INICO. Universidad de Valladolid.
} 
Evaluación de la calidad de vida en personas mayores y con discapacidad: la Escala Fumat

\section{ABSTRACT}

The aim of this article is to develop and validate a specific tool to assess objective quality of life in elder people and people with disabilities who are social services recipients. The scale was developed in three steps: literature review, development of a pool of items, and panel of 15 experts evaluating the items idoneity, importance, and observability. The questionnaire validation was carried out with a sample composed of 100 adult users of services provided by Matia Fundazioa (San Sebastian, Spain). Reliability was evaluated in terms of internal consistence (Cronbach's Alpha and Spearman-Brown splithalf reliability) and factorial structure (theta and omega reliability). Validity was evaluated in terms of content, discriminant, concurrent and construct validity. The obtained results confirm the FUMAT Scale validity and reliability to assess objective quality of life in elder people and persons with disabilities that are users of social services.

\section{KEY WORDS} rics.

Quality of life assessment, elderly, Disability, Assessment, Measurement, Psychomet-

\section{AGRADECIMIENTOS}

La realización de este trabajo ha sido posible gracias a la financiación y a la colaboración de Matia Fundazioa, con motivo de la I Beca Sociosanitaria Matia Fundazioa Memorial Peli Egaña. Los autores agradecen especialmente a los jueces sus valiosas contribuciones en el desarrollo de la Escala FUMAT y a los profesionales que la cumplimentaron por su esfuerzo y dedicación. 
España es uno de los países europeos que ha experimentado el proceso de envejecimiento demográfico a un ritmo más rápido. Es un hecho constatado que en los últimos años ha aumentado de forma considerable el número de personas que llegan a la vejez, y entre ellas también el número de personas con discapacidad que llegan a edades avanzadas. El descenso de la natalidad, el aumento de la esperanza de vida, los avances en las ciencias de la salud y la mejora de las condiciones de vida son algunas de las causas que han propiciado esta situación. A pesar de que la expresión 'envejecimiento demográfico' se utiliza a menudo con connotaciones negativas e incluso catastrofistas, alude a aspectos positivos como la mayor esperanza de vida. No obstante, ha planteado un nuevo reto y provocado un fuerte impacto en las políticas sociales, en los intereses y prácticas profesionales, y en el diseño y la provisión de servicios y apoyos, para los cuales es importante no solo una vida más longeva sino una vida de calidad. Los esfuerzos hoy van dirigidos a añadir vida a los años, más que a añadir años a la vida (Clark, 1995).

La presente investigación se basa en la urgente necesidad planteada desde los servicios sociales dirigidos a personas mayores y personas con discapacidad de contar con instrumentos que permitan la evaluación de la calidad de vida de sus usuarios, desde un enfoque consensuado y práctico para la intervención y la mejora de resultados personales. En la actualidad contamos con instrumentos que permiten la evaluación de la calidad de vida de personas mayores; sin embargo, la mayoría de estos evalúan la calidad de vida relacionada con la salud sin tener en cuenta otros aspectos importantes de la vida de las personas. En cuanto a la evaluación de personas con discapacidad, existen instrumentos novedosos, sobre todo en el campo de la discapaci- dad intelectual, si bien la mayoría están dirigidos a adultos $\mathrm{y}$, aunque son aplicables también a personas mayores, no tienen en cuenta las peculiaridades de esta etapa de la vida. El objetivo de este estudio no es construir un instrumento específico para personas mayores ni para personas con discapacidad, sino una herramienta válida para todas ellas. Tampoco el objetivo es contar con una escala de carácter subjetivo y basada en la percepción de la persona evaluada, sino realizar una evaluación objetiva de la calidad de vida, que sea sensible a los cambios en el modo de proporcionar los servicios y los apoyos, en su organización, y en las actividades de mejora que se puedan planificar; esto es, un instrumento que cuente con items objetivos y observables por un observador externo, pues de acuerdo con la investigación son los que pueden ser sensibles a los cambios (Schalock y Felce, 2004; Verdugo, Schalock, Gómez y Arias, 2007; Verdugo, Schalock, Keith y Stancliffe, 2005).

En este sentido, encontramos en español sólo dos instrumentos de reciente publicación que sirvan para la evaluación objetiva de calidad de vida: la Escala Integral (Gómez, 2005; Verdugo, Arias y Gómez, 2006; Verdugo, Arias, Gómez y Schalock, 2009; Verdugo, Gómez y Arias, 2007) y la Escala GENCAT (Gómez, Verdugo y Arias, 2007; Verdugo, Arias, Gómez y Schalock, 2008a, 2008b). Descartamos el uso de la Escala Integral por ir dirigida a una población demasiado especifica: personas adultas con discapacidad intelectual. La Escala GENCAT se descartó por el motivo contrario, no ser específica para población objetivo ya que está dirigida a usuarios de servicios sociales en general. Dada la inexistencia de una escala objetiva de calidad de vida específica y adaptada a las peculiaridades de las personas mayores -con discapacidad o sin ella- y útil también para personas con discapacidad -mayores 0 
no-, se planteó el desarrollo de la Escala FUMAT.

Con el fin de evitar la inadecuada y confusa utilización de la terminologia que es característica de la investigación sobre el binomio envejecimiento-discapacidad, los términos genéricos de personas con discapacidad y personas mayores implicados en esta investigación se concretan más para hacer referencia a un numeroso colectivo de personas que pueden agruparse bajo los siguientes epígrafes (Aguado y Alcedo, 2004; Aguado, Alcedo, Fontanil, Arias y Verdugo, 2006; Verdugo, 2000):

Personas con discapacidad. La Encuesta sobre discapacidades, deficiencias y estado de salud (INE, 2000) en España aporta datos epidemiológicos sobre 3.528.221 personas con discapacidad, lo que supone un $9 \%$ de la población general.

Personas con discapacidad que envejecen. Hace referencia a aquellas personas con discapacidad que envejecen con ella y todas sus implicaciones (Aguilella, Verdugo y Sánchez, en prensa).

Personas que envejecen y adquieren una discapacidad. Personas que envejecen y de forma natural adquieren alguna discapacidad propia de la edad, como consecuencia del propio envejecimiento. Este grupo y el anterior pueden combinarse en uno solo bajo el epígrafe personas mayores con discapacidad, que englobaria a 2.072.672 personas, esto es, el $58,74 \%$ del total de personas con discapacidades, y un $32,21 \%$ de la población sin discapacidad en ese grupo de edad.

Personas mayores. Personas que envejecen sin presentar una discapacidad relevante.
Una vez definidas las poblaciones, el siguiente concepto fundamental a definir es el término calidad de vida. En esta investigación se aborda la evaluación de la calidad de vida de los colectivos anteriormente mencionados desde un marco teórico común -el modelo de Schalock y Verdugo $(2002 / 2003)$, ya que ha sido el más citado internacionalmente en el ámbito de la discapacidad intelectual en los últimos años y se está extendiendo en nuestros días a otros colectivos y al ámbito de los servicios sociales en general. Según este modelo (Brown, Keith y Schalock, 2004; Schalock y Verdugo, 2007; Verdugo, 2006), calidad de vida individual es un estado deseado de bienestar personal que: (a) es multidimensional; (b) tiene propiedades etic y emic (Jenaro et al., 2005; Schalock et al., 2005); (c) tiene componentes objetivos y subjetivos; (d) está influenciada por factores personales y ambientales; y (e) incorpora la perspectiva de sistemas que engloba los múltiples ambientes que influyen en la vida de las personas (Keith y Bonham, 2005; Schalock, Gardner y Bradley, 2007; Schalock, Bonham y Verdugo, 2008; Schalock, Verdugo, Bonham, Fantova y van Loon, 2008).

Además de por su extendido uso en distintos ámbitos, de la gran aceptación en la comunidad científica internacional y de sus múltiples aplicaciones prácticas, la elección de este modelo de calidad de vida para el desarrollo del presente estudio se ha basado en la constatación de que el modelo se ha desarrollado siguiendo tres pasos (Schalock y Verdugo, 2007): observar y describir el fenómeno estudiado, trasladarlo a un mapa conceptual y evaluar el modelo. En este momento, nos encontramos en el inicio de la fase consistente en desarrollar una teoría de calidad de vida (Schalock et al., en prensa). Como ha quedado patente, se puede afirmar que nos encontramos en el momento idóneo 
para iniciar la aplicación del concepto en la política social planificada, pues este marco teórico se ha convertido en una referencia para la prestación de servicios, un fundamento para las prácticas basadas en la evidencia y un vehículo para desarrollar estrategias de mejora de la calidad (Schalock, Bonham y Verdugo, 2008; Schalock y Verdugo, 2002/2003, 2007; Schalock, Gardner y Bradley, 2007).

\section{PRIMER ESTUDIO: DESARROLLO DE LA ESCALA}

Se siguió un riguroso y sistemático proceso para el desarrollo de la escala, siguiendo las recomendaciones para la construcción de escalas de calidad de vida multidimensionales centradas en el contexto (Verdugo et al., 2007; Schalock et al., en prensa). Como se ha comentado anteriormente, el marco teórico en el que se sustentó el desarrollo de la escala fue el planteado por Schalock y Verdugo (2002/2003). El objetivo del instrumento es evaluar de forma objetiva la calidad de vida de personas con discapacidad y personas mayores usuarias de algún tipo de servicio social. A partir de dicho marco y una vez definido el objetivo de la evaluación y concretada la población a la que se dirige el instrumento, se llevó a cabo una exhaustiva revisión de la literatura científica que permitió la elaboración de un banco de 300 ítems con formato declarativo, enunciados en tercera persona para ser contestados con una escala de frecuencia y que se organizaron de acuerdo según las dimensiones e indicadores de calidad de vida (Tabla 1). Entre éstos, se seleccionaron 18-20 items para cada una de las ocho dimensiones $(\mathrm{N}=158)$.

Tabla 1. Definición operativa de calidad de vida

\begin{tabular}{|c|c|}
\hline DIMENSIONES & INDICADORES \\
\hline \multirow[t]{2}{*}{ AUTODETERMINACIÓN(ADT) } & $\begin{array}{l}\text { Autonomia; Decisiones; Elecciones; Metas y } \\
\text { preferencias personales }\end{array}$ \\
\hline & Conocimiento de derechos; Ejercicio de \\
\hline \multirow[t]{2}{*}{ DERECHOS (DER) } & $\begin{array}{l}\text { derechos; Defensa de derechos; Información; } \\
\text { Respeto; Intimidad; Responsabilidades; }\end{array}$ \\
\hline & $\begin{array}{l}\text { Dignidad; Derechos Legales } \\
\text { Estabilidad emocional (salud mental): }\end{array}$ \\
\hline \multirow{2}{*}{ BIENESTAR EMOCIONAL (BE) } & Ausencia de estrés y sentimientos negativos; \\
\hline & Autoconcepto; Satisfacción \\
\hline \multirow[t]{2}{*}{ INCLUSIÓN SOCIAL (IS) } & Integración; Participación; Apoyos \\
\hline & Educación, Oportunidades de aprendizaje; \\
\hline \multirow[t]{2}{*}{ DESARROLLO PERSONAL (DP) } & $\begin{array}{l}\text { Habilidades funcionales; Habilidades } \\
\text { académicas; }\end{array}$ \\
\hline & Relaciones de pareja; Relaciones familiares; \\
\hline \multirow[t]{2}{*}{ RELACIONES INTERPERSONALES (RI) } & $\begin{array}{l}\text { Relaciones Sociales; Tiene amigos claramente } \\
\text { identificados; contactos sociales positivos y } \\
\text { gratificantes }\end{array}$ \\
\hline & Ingresos; Posesiones; Condiciones de la \\
\hline \multirow[t]{2}{*}{ BIENESTAR MATERIAL (BM) } & Trabajo;Condiciones del servicio; Empleo; \\
\hline & Salud General; Atención Sanitaria; Memoria; \\
\hline \multirow[t]{2}{*}{ BIENESTAR FÍSICO (BF) } & $\begin{array}{l}\text { Dolor; Sueño; Visión; Audición; Movilidad; } \\
\text { Mediación; Acceso a ayudas técnicas; }\end{array}$ \\
\hline & Actividades de la vida diaria. \\
\hline
\end{tabular}


Evaluación de la calidad de vida en personas mayores y con discapacidad: la Escala Fumat

Con el fin de comprobar la validez de contenido de los ítems seleccionados, fueron sometidos a la valoración de un grupo de jueces expertos. Los objetivos concretos de la consulta fueron comprobar: (a) si los items eran idóneos e importantes para medir la calidad de vida de los colectivos implicados; (b) si los items eran observables; y (c) si los principales indicadores de calidad de vida estaban representados o si, por el contrario, se había obviado algún indicador importante.

\section{MÉTODO}

\section{Participantes}

El grupo de jueces estuvo formado por un total de 15 expertos. La mayoría $(86,7 \%)$ de los expertos fueron mujeres. De ellos, $10(66,67 \%)$ eran expertos en el colectivo de personas mayores y 5 en el colectivo de personas con discapacidad (33,33\%). El 80\% tenía más de 5 años de experiencia en atención especializada.

\section{Procedimiento}

El contacto y la solicitud de participación a los jueces se realizaron por correo electrónico. Junto con la carta de solicitud de participación, se envió a los expertos una hoja para que rellenaran algunos datos personales, un documento con las instrucciones para completar la tarea y la plantilla con los items que debían valorar. Su tarea consistió en valorar cada uno de los ítems en una escala de 1 a 6 en los tres criterios apuntados anteriormente: idoneidad, importancia y observabilidad. Además se les dio la posibilidad de añadir tres ítems 0 indicadores para cada una de las dimensiones de calidad de vida, que consideraran importantes y que no estuvieran incluidos. Del mismo modo, se les solici- tó que incluyeran todos los comentarios que consideraran oportunos.

\section{RESULTADOS}

Para retener un item y que éste formara parte de la versión inicial de la Escala FUMAT, se tuvieron en cuenta los siguientes criterios: (a) la media del item debía superar 5 puntos con una desviación típica inferior a 2,5 puntos en idoneidad; y (b) la media debía ser superior a 4 y la desviación típica inferior a 3 en importancia y observabilidad. Tan sólo 10 items no cumplieron los requisitos establecidos para la idoneidad. De éstos, 8 items además no cumplieron los requisitos referidos a la importancia y 6 no cumplieron los de observabilidad. Por ello, los 10 items fueron eliminados, quedando un total de 148 ítems.

A continuación se procedió a determinar la consistencia del juicio de los expertos, a través del coeficiente Alfa de Cronbach para la idoneidad, importancia y observabilidad de todos los ítems. Los coeficientes obtenidos indicaron una alta correlación entre cada juez y el conjunto en el caso de la idoneidad y la importancia $(\alpha>, 725)$ y ligeramente más baja en el caso de la observabilidad $(\alpha>, 657)$. En cuanto a las puntuaciones promedio otorgadas a los ítems en cada una de las categorías, los jueces expertos asignaron una calificación muy alta a todos los items en idoneidad $(\mathrm{M}=4,98 ; \mathrm{DT}=$ $1,35)$, y observabilidad $(\mathrm{M}=4,86$; $\mathrm{DT}=$ $0,36)$, pero especialmente en importancia $(\mathrm{M}=5,05$; $\mathrm{DT}=0,40)$. Confirmada la adecuación de los 148 ítems restantes, a continuación el equipo de investigación seleccionó entre ellos los 80 más adecuados para formar parte de la Escala FUMAT; esto es, un total de 10 ítems por cada una de las ocho dimensiones de calidad de vida con la condición de contar al menos con un item para cada uno 
de los indicadores de calidad de vida que las componen.

\section{SEGUNDO ESTUDIO: VALIDACIÓN DE LA ESCALA}

La versión piloto de la Escala FUMAT se aplicó a una muestra de 100 personas mayores y con discapacidad con el objetivo de: (a) estudiar las propiedades psicométricas de la versión piloto del instrumento; (b) eliminar los items menos adecuados en función de su fiabilidad; (c) analizar la fiabilidad y la validez de la versión final; y (d) proporcionar baremos que permitan interpretar las puntuaciones de calidad de vida obtenidas.

\section{MÉTODO}

\section{Participantes}

En la validación de la Escala FUMAT participaron 100 usuarios de los 5 centros de Matia Fundazioa: Centro de Atención Residencial Especializada IZA (20\%), Julián Rezola (20\%), Lamourous (20\%), Fraisoro (20\%9 y Txara $1(20 \%)$. De éstos, los cuatro últimos son centros residenciales para personas mayores, mientras que el primero es un centro para personas con discapacidad. De este modo, el $80 \%$ de la población evaluada fueron personas mayores mientras el $20 \%$ restante fueron personas con discapacidad. La mayoria de los participantes fueron mujeres (56\%). El rango de edades osciló entre 24 y 99 años, siendo la media 74,67. El grupo de edad minoritario fue el de personas menores de 65 años, que suponia un $24 \%$ de la muestra total. Más del 50\% de la muestra tenía una edad superior a 78 años.

Los profesionales que cumplimentaron las Escalas fueron nueve. Todos excepto uno eran mujeres. La mayoría de las escalas fueron completadas por cinco psicólogos $(85,74 \%)$, un porcentaje considerable $(10,2 \%)$ fueron completadas por trabajadores sociales y el resto (4\%) por un terapeuta ocupacional. Las edades de los evaluadores estaban comprendidas entre los 25 y los 36 años.

\section{Instrumento}

La versión piloto de la Escala FUMAT se aplicó de forma electrónica. Como se ha mencionado, este instrumento proporciona un perfil objetivo de la calidad de vida de los usuarios adultos de servicios sociales. Se trata de un cuestionario autoadministrado en el que el profesional responde a cuestiones observables sobre la calidad de vida del usuario. El tiempo de cumplimentación oscila entre 10 y 15 minutos. Está compuesta por un total de 80 items, enunciados en tercera persona, con formato declarativo, y ordenados de forma aleatoria dentro de su correspondiente dimensión. Todos los items pueden responderse con un formato de respuesta de frecuencia de cuatro opciones ('nunca o casi nunca', 'a veces', 'frecuentemente', 'siempre o casi siempre'). No obstante, para aquellos items a los que resulta difícil contestar con esta escala de frecuencia, se da la instrucción de responder pensando en la siguiente escala Likert de cuatro puntos ('totalmente de acuerdo', 'de acuerdo', 'en desacuerdo', 'totalmente en desacuerdo'). El cuestionario fue diseñado para generar un indice global de calidad de vida, así como para proporcionar puntuaciones en cada una de las dimensiones y un perfil de la persona.

\section{Procedimiento}

En primer lugar se envió un correo electrónico explicando los objetivos del estudio y solicitando la participación a 
los profesionales. En este correo se les indicaba la dirección Web donde podian encontrar la versión electrónica de la Escala FUMAT, el manual con las instrucciones necesarias para completar la escala de forma autónoma, la descripción del proyecto de investigación, una breve descripción del concepto de calidad de vida y una detallada explicación del procedimiento de aplicación. Se les proporcionó, además, una dirección de correo electrónico para que pudieran realizar las consultas, comentarios 0 sugerencias que estimaran oportunos. La aplicación de la escala tuvo lugar en septiembre de 2007.

\section{RESULTADOS}

\section{Análisis estadistico y eliminación de los items}

En primer lugar, se realizó un estudio de las propiedades psicométricas de los items con el fin de conocer su poder discriminativo y su contribución a la homogeneidad del test. Para ello, se analizó la consistencia interna inicial de cada una de las subescalas o dimensiones de calidad de vida mediante el coeficiente Alfa de Cronbach y la correlación entre dos mitades de Spearman-Brown. La versión piloto de la Escala FUMAT obtuvo una consistencia interna inicial bastante alta $(\alpha=, 954 ; r=, 905)$. A continuación, se analizó la fiabilidad de los items mediante el Índice de Homogeneidad Corregida $\left(\mathrm{IH}_{\mathrm{c}}\right)$.

Se eliminaron 23 items por contribuir su eliminación a aumentar la consistencia interna de la respectiva dimensión. De estos 23, se eliminaron además de por esta razón, 11 items por tener un $\mathrm{IH}_{\mathrm{c}}$ inferior a ,300 (los items 5 y 9 pertenecientes a Bienestar emocional; 11, 14, 17 y 20 de Relaciones interpersonales; 31 y 40 de Desarrollo personal; 52 y 54 de
Autodeterminación; y 65 de Inclusión social). Por tener un $\mathrm{IH}$ inferior a ,200, se eliminaron 9 ítems (los items 41, 43, 47 y 48 de Bienestar fisico; 72, 73, 75, 76 y 79 de Derechos). Finalmente, por tener un $\mathrm{IH}$ inferior a , 100 se eliminaron 3 items $(24,25$ y 29 de Bienestar material).

\section{Propiedades Psicométricas}

Tras el proceso de eliminación de items, la consistencia interna aumentó ligeramente según el coeficiente Alfa de Cronbach $(\alpha=, 962)$ y disminuyó levemente según la correlación entre dos mitades de Spearman-Brown $(r=$,894). No obstante, podemos concluir que la escala presenta una fiabilidad bastante elevada aun si se acorta considerablemente la longitud de la escala (de 80 a 57 items). En cuanto a la fiabilidad de las ocho dimensiones de calidad de vida, la eliminación de ítems dio lugar a un considerable incremento de la consistencia interna de todas ellas. Como se muestra en la Tabla 2, se encontraron coeficientes de consistencia interna bastante elevados, excepto en el caso de la dimensión Bienestar material $(\alpha=, 694$; $\mathrm{r}=, 308$ ), que mostró una consistencia adecuada según el coeficiente de alfa de Cronbach pero muy baja en el caso del coeficiente de Spearman Brown. El resto de las dimensiones presentaron coeficientes superiores a ,700. La dimensión que presentó mayor consistencia interna fue Desarrollo personal, seguida de Autodeterminación, con coeficientes superiores a ,900.

Como complemento a los índices de consistencia interna, se presentan en la misma tabla otros dos indicadores de la fiabilidad basada en la estructura factorial de la escala: Zeta $(\theta)$ (Carmines y Zeller, 1979) y Omega $(\Omega)$ (Heise y Bohrnstedt, 1970). Ambos coeficientes son estimadores del limite superior de $\alpha$, 
Tabla 2. Coeficientes de fiabilidad de las subescalas

\begin{tabular}{|lccccc|}
\hline ESCALA FUMAT & $\begin{array}{c}\text { Alpha de } \\
\text { Cronbach }\end{array}$ & $\begin{array}{c}\text { Dos } \\
\text { mitades }\end{array}$ & - & - & N Items \\
\hline BIENESTAR EMOCIONAL &, 860 &, 901 &, 868 &, 967 & 8 \\
RELACIONES &, 807 &, 753 & & & 6 \\
INTERPERSONALES & & &, 821 &, 933 & 7 \\
BIENESTAR MATERIAL &, 604 &, 308 &, 626 &, 893 & 8 \\
DESARROLLO PERSONAL &, 918 &, 928 &, 936 &, 953 & 6 \\
BIENESTAR FISICO &, 732 &, 781 &, 744 &, 909 & 8 \\
AUTODETERMINACIÓN &, 908 &, 845 &, 915 &, 950 & 8 \\
INCLUSIÓN SOCIAL &, 862 &, 870 &, 864 &, 939 & 9 \\
DERECHOS &, 774 &, 823 &, 794 &, 873 & 5 \\
ESCALA TOTAL &, 962 &, 894 &, 968 &, 991 & 57 \\
\hline
\end{tabular}

siendo su ordenación en lo referente a su cuantía como sigue: $\alpha<\theta<\Omega$. Los coeficientes $\emptyset$ de las subescalas 0 dimensiones oscilaron entre ,873 y ,967. Las dimensiones menos fiables fueron Derechos y Bienestar material. La escala total mostró un coeficiente $\Omega=, 991$ por lo que se puede afirmar que la prueba tiene una fiabilidad muy alta según este coeficiente. En cuanto a los coeficientes $\theta$, oscilaron entre ,626 y ,936. La dimensión que mostró menor fiabilidad fue Bienestar material, seguida de Bienestar fisico y Derechos. La escala total mostró un coeficiente $\Omega=, 968$, redundando en la alta fiabilidad de la escala.

Por otra parte, a fin de comprobar la capacidad de la escala para diferenciar entre sujetos con diferentes niveles globales de calidad de vida, se llevó a cabo un análisis cluster de k-medias a partir de las puntuaciones obtenidas en las ocho dimensiones. El análisis mostró la existencia de tres grupos diferenciados. El cluster 1 (37 casos) obtuvo puntuaciones medias en seis de las ocho dimensiones, y más altas en Bienestar Emocional y Bienestar Material. En estas dos dimensiones, junto con Relaciones Interpersonales, no se observaron diferencias significativas con respecto al cluster 3, conformado por los 49 casos que alcanzaron las puntuaciones más elevadas en el conjunto de los factores. El resto de los sujetos se agrupan en el cluster 2 , y presentan un perfil de puntuaciones más bajas en todas las dimensiones con excepción del Bienestar Material. Una vez configurados los clusters como se ha descrito, se procedió a realizar un análisis discriminante múltiple tomando las puntuaciones en las respectivas dimensiones como variables independientes y la pertenencia a los clusters mencionados como variable de agrupación. Los resultados $\left(\lambda\right.$ de Wilks $=.064 ; \mathrm{F}_{(16,180)}=$ 33,$26 ; \mathrm{p}=, 000$ ) pusieron de manifiesto la capacidad de la función para clasificar correctamente al $100 \%$ de los participantes de los clusters 2 y 3 , y al $94,59 \%$ de los participantes del cluster 1 .

\section{CONCLUSIONES}

A la vista de estos datos, se puede concluir que la Escala FUMAT supone una valiosa aportación a la investigación sobre calidad de vida y, especialmente, al campo aplicado de la intervención en personas mayores y personas con discapacidad. El instrumento permite evaluar en pocos minutos y de una forma muy sencilla la calidad de vida de estas poblaciones cuando son usuarias de algún tipo de servicio social o clientes de algún tipo de entidad u organización que tenga entre sus objetivos la mejora de la calidad de vida. De este modo, la escala 
surge como un instrumento válido y fiable para la planificación de apoyos individualizados e intervenciones centradas en la persona, así como para encaminar las intervenciones dirigidas a la mejora de la calidad de la organización y la entidad.

El tamaño y la escasa representatividad de la muestra pueden señalarse como limitaciones fundamentales del estudio. No obstante, futuras líneas de investigación tienen como objetivos principales: (a) confirmar las propiedades psicométricas del instrumento en una muestra más amplia y perteneciente a distintas entidades de distintas comunidades autónomas; (b) estudiar la validez concurrente del instrumento mediante el análisis de la correlación entre la Escala FUMAT y otro instrumento que mida el mismo constructo; y (c) analizar la validez de constructo a través de la realización de un estudio factorial confirmatorio. A pesar de dichas limitaciones y, teniendo en cuenta de que el aquí descrito es un estudio preliminar, podemos concluir que es un instrumento con propiedades adecuadas y criterios válidos que justifican su uso y aplicación en la práctica aplicada y en el ámbito de la investigación.

\section{BIBLIOGRAFÍA}

Aguado, A. L. y Alcedo, M. A. (2004). Necesidades percibidas en el proceso de envejecimiento de las personas con discapacidad. Psicothema, 16(2), 261-269.

Aguado, A. L., Alcedo, M. A., Fontanil, Y., Arias, B. y Verdugo, M. A. (2006). Calidad de vida y necesidades percibidas en el proceso de envejecimiento de las personas con discapacidad. Manuscrito sin publicar.

Aguilella, A., Verdugo, M. A. y Sánchez, M. C. (en prensa). Calidad de vida familiar y apoyos para los progenitores de personas con discapacidad intelectual en proceso de envejecimiento. Siglo Cero.

Brown, I., Keith, K. D., y Schalock, R. L. (2004). Quality of life conceptualization, measurement, and application: Validation of the SIRG-QOL consumer principles. Journal of Intellectual Disability Research, 48(4 y 5), 451.

Carmines, E. y Zeller, R. (1979). Reliability and Validity Assessment. Newbury Park, CA: Sage PublicationsClark, 1995.
Gómez, L. E. (2005). Construcción de una escala de calidad de vida para adultos con discapacidad intelectual. Trabajo de grado sin publicar, Universidad de Salamanca, Salamanca.

Gómez, L. E., Verdugo, M. A. y Arias, B. (2007). Aplicación del Modelo de Calidad de Vida en los Servicios Sociales de Cataluña. INTEGRA, 10 (27), $4-5$.

Heise, D. R. y Bohrnstedt, G. W. (1970). Validity, invalidity and reliability. En: Borgatta, E. F. y Bohrnstedt, G. W. (Eds.), Sociological Methodology (pp. 104-129). San Francisco: Jossey-Bass.

INE (2000). Encuesta sobre discapacidades, deficiencias y estados de salud: Año 1999. Avances de resultados. Madrid: Instituto Nacional de Estadística.

Jenaro, C., Verdugo, M. A., Caballo, C., Balboni, G., Lachapelle, Y., Otbrebski, W. y Schalock, R. L. (2005). Cross-cultural study of person-centered quality of life domains and indicators: A replication. Journal of Intellectual Disability Research, 49, 734-739. 
Keith, K. D., y Bonham, G. S. (2005). The use of quality of life data at the organization and systems level. Journal of Intellectual Disability Research, 49(10), 799-805.

Schalock, R. L., Bonham, G. S. y Verdugo, M. A. (2008). The concept of quality of life as a framework for program planning, implementation, evaluation, and improvement. Evaluation and Program Planning, 26(3), 229-235.

Schalock, R. L., Gardner, J. F., y Bradley, V. J. (2007). Quality of life of persons with intellectual and other developmental disabilities: Applications across individuals, organizations, communities, and systems. Washington, DC: American Association on Intellectual and Developmental Disabilities.

Schalock, R. L. y Felce, D. (2004). Quality of life and subjective well-being: conceptual and measurement issues. En E. Emerson, C. Hatton, T. Thompson, y T.R. Parmenter (Eds.), International handbook of applied research in intellectual disabilities (pp. 261-279). London: John Wiley and Sons.

Schalock, R. L., Keith, K., Verdugo, M. A. y Gómez, L. E. (en prensa). Quality of life theory construction and model development. Journal of Intellectual Disability Research.

Schalock, R. L. y Verdugo, M. A. (2002/2003). Quality of life for human service practitioners. Washington, DC: American Association on Mental Retardation [Traducido al castellano por M.A. Verdugo y C. Jenaro. Calidad de vida. Manual para profesionales de la educación, salud y servicios sociales. Madrid: Alianza].

Schalock, R. L. y Verdugo, M. A. (2007). El concepto de calidad de vida en los servicios y apoyos para personas con discapacidad intelectual. Siglo Cero, 38(4), 21-36.

Schalock, R. L. y Verdugo, M. A. (2008). Quality of life conceptual and measurement frameworks: from concept to application in the field of intellectual disabilities. Evaluation \& Program Planning, 31(2), 181-190.

Schalock, R. L., Verdugo, M. A., Bonham, G.S., Fantova, F. y Van Loon, J. (2008). Enhancing personal outcomes: organizational strategies, guidelines, and examples. Journal of Policy and Practice in Intellectual Disabilities, 5(1), 18-28.

Schalock, R. L., Verdugo, M. A., Jenaro, C., Wang, M., Wehmeyer, M., Xu, J. y Lachapelle, Y.
(2005). A cross-cultural study of quality of life indicators. American Journal on Mental Retardation, $110,298-311$

Verdugo, M.A. (2000). Calidad de vida en el envejecimiento de las personas con discapacidades intelectuales y del desarrollo. En P. Rodríguez, A. Aguado y A. Carpintero (Coord.) Discapacidad y Envejecimiento. Madrid: Escuela Libre Editorial.

Verdugo, M. A. (2006). Cómo mejorar la calidad de vida de las personas con discapacidad. Salamanca: Amarú

Verdugo, M. A., Arias, B. y Gómez, L. E. (2006). Escala integral de medición subjetiva y objetiva de la calidad de vida en personas con discapacidad intelectual. En M.A. Verdugo (Dir.), Cómo mejorar la calidad de vida de las personas con discapacidad. Instrumentos y estrategias de evaluación (pp. 417-448). Salamanca: Amarú.

Verdugo, M. A., Arias, B., Gómez L. E. y Schalock, R. L. (2008a). Formulari de l'Escala Gencat de Qualitat de vida. Manual d'aplicació de l?Escala Gencat de Qualitat de vida. Departamento de Acción Social y Ciudadanía. Generalitat de Cataluña.

Verdugo, M. A., Arias, B., Gómez L. E. y Schalock, R. L. (2008b). Escala Gencat. Informe sobre la creació d'una escala multidimensional per avaluar la qualitat de vida de les persones usuàries dels serveis socials a Catalunya. Departamento de Acción Social y Ciudadanía. Generalitat de Cataluña.

Verdugo, M. A., Arias, B., Gómez, L. E. y Schalock, R. L. Escala Integral de Calidad de Vida. Madrid: CEPE.

Verdugo, M. A., Gómez, L. E. y Arias, B. La Escala Integral de calidad de vida. Desarrollo y estudio preliminar de sus propiedades psicométricas.

Verdugo, M.A., Schalock, R. L., Gómez L.E. y Arias, B. (2007). Construcción de escalas de calidad de vida multidimensionales centradas en el contexto: la Escala GENCAT Siglo Cero, 38(4), 5772.

Verdugo, M. A., Schalock, R. L., Keith, K. D. y Stancliffe, R. (2005). Quality of life and its measurement: important principles and guidelines. Journal of Intellectual Disability Research, 49(10), 707717. 